\title{
Physical Properties of Fiber-Optic Sensor in New Dual Configuration
}

M. Życzkowski*, M. Szustakowski, P. Markowski And M. Karol Institute of Optoelectronics, Military University of Technology

S. Kaliskiego 2, 00-908 Warsaw, Poland

The main aim of the work is the integration of two different fiber-optic sensors: the Michelson interferometric sensor, the Mach-Zehnder interferometric, the Sagnac interferometric and modalmetric sensors. The proposed hybrid sensor is a classical interferometer whose measuring arm is upgraded with modalmetric sensor. The theme of research is to assess the impact of modalmetric sensor to interferometer and comparative assessment of detection capabilities of interferometric sensor, modalmetric sensor and hybrid sensor for different types of excitations. The described tests concern the area of basic researches. The results can be used in the construction sensors with corresponding characteristics and properties to the appropriate applications.

PACS: 42.30.Wb, 87.57.-s, 42.79.Qx

\section{Interferometric sensors}

Fiber-optic interferometric sensors are included to group of phase sensors [1-4]. As a result of effects mechanical external field on fiber is a change optical path length for propagating in this piece of fiber electromagnetic wave. This causes a phase change a wave propagating in the measurement fiber in relation to a wave propagating in the reference fiber according to the relation

$$
\Delta \Phi=\frac{2 \pi}{\lambda_{0}} \Delta L,
$$

where $\Delta \Phi$ - phase shift, $\Delta L$ - optical path length shift, $\lambda_{0}$ - wavelength.

The difference of these phases at the output of the fiber reflects the change field acting on the fiber. The interferometric sensor systems, phase difference, or actually change of the intensity of the interference image is read on the detector. If the optical path for both arms of the interferometer is the same and there is no influence of the external field as a result of constructive interference at the output is maximum light intensity. At the time of occurrence of the impact of the external field on one branch, the output waves arrive at different phases, and as a result of destructive interference the light intensity is respectively lower. For the case when both waves reach in the counter phase there will be complete extinction of light.

The intensity of radiation at the output of the interferometer can be described by the formula [1]:

$$
I_{\mathrm{OUT}}=\left\langle E E^{*}\right\rangle,
$$

where

$$
E=E_{1}+E_{2} .
$$

$E_{1}, E_{2}$ - the vector amplitude of light propagating in the first and second arm of the interferometer.

For interferometer systems output signal depends on the response characteristics of the system. Frequency response depends on factors such as:

\footnotetext{
* corresponding author; e-mail: mzyczkowski@wat.edu.pl
}

- The parameters of the light source,

- Division of fiber-optic coupler,

- Imbalance optical path of interferometer arms.

In most cases we are able to shape the characteristics of the interferometric system to obtain the properties of the assumed final parameters. This is achieved through the selection of sources of radiation, integrated passive optical paths, configuration of the sensor and matching circuits within the meaning of electronic integrated demodulator output signals. It turns out that the configurations obtained composition are not ideal solutions. For example, interferometric sensors are too sensitive systems, in terms of detection of the desired field and the signal representing the ambient noise. They reflect abnormal signal as a signal of constant component amplitude transformed by the characteristics of the system, as for the signal response analysis ranks them in a group of sensors that require complex detection methods.

\section{Modalmetric sensor}

Modalmetric sensor is a system built from the light source and a detector which is located between the transmission channel consists of two segments of optical fibers between which there is a section of multimode fiber. Sensitive element is a piece of multimode fiber. The radiation emanating from the source through a single-mode fiber feeds core multimode fiber. The output radiation of multimode fiber illuminates the face of single-mode fiber. Single-mode fiber "sees" only a fragment of cross-field multimode fiber output. As a result of external influence, such as a mechanical disorder of the multimode section, it changes the field distribution propagating electromagnetic wave. At the output, multimode fiber is seen as the spatial change of the transverse distribution of intensity interference between the modal products, groups of modes. This change is attributable to changes in the way of propagation, optical paths for different modes, modes groups under influence of external mechanical fields.

Intensity distribution of radiation in a multimode optical fiber can be described by the formula [3]: 


$$
\begin{aligned}
& I(x, y, z)=\lim _{T \rightarrow \infty} \frac{1}{T} \int_{-\frac{T}{2}}^{\frac{T}{2}}|u(x, y, z ; t)|^{2} \mathrm{~d} t \\
& \quad=|A(x, y, z)|^{2},
\end{aligned}
$$

where $x, y, z-$ geometric dimensions of the fiber.

Therefore, the radiation introduced into the single-mode optical fiber with multimode fiber, is changed depending on the externalities. The forehead of single-mode fiber is the "specific" diaphragm, through which the head of multimode fiber is observed by single mode fiber and transmits, limited to the dimensions of the numerical aperture, the signal to the detector. Changing the intensity of radiation incident on the detector is proportional to the disorder in a multimode fiber. However, it is converted by the frequency response characteristic of the sensor.

The system characteristics are influenced by:

- Parameters of the source,

- The length of multimode fiber sensing,

- The length of pieces of single-mode optical fiber from the detector,

- Parameters of the detector,

- The applicable sections of connection types between multimode and single-mode optical fibers.

In order to improve modalmetric system sensitivity, it seeks to suppress cladding modes in single-mode fiber. This can be achieved through modal traps or by fit of a reasonably long section of single mode fiber. This is to eliminate higher-order modes of transition, with a multimode fiber to single mode, whose energy would be added together on the detector and be a noise. In such systems it should been sought that to the detector came the light falling only from single-mode fiber core area. Then, modalmetric system is a system of responding to micro-bending mechanical and physical strain. The output signal from the modalmetric sensor does not reflect, in the metrological sense, disorders of the signals. It is a sensor that responds to the fact that there are occurrence, and its response to the signal loss is revealed in a random way as to the amplitude and frequency characteristics. Therefore, its main applications are security systems, which is only important response to the phenomenon of the sensor system.

\section{Dual sensor - motivation research}

This article describes the use of three optical interferferometers of classic design, for which in the measuring circuit multimode fiber was attached instead single mode fiber. The aim is to attach to the integrated structure of the interferometer sensor the structure of modalmetric sensor. Using the dual configuration is to find new properties of fiber-optic sensor detection application attractive. As mentioned earlier, interferometric sensors have high sensitivity and their response amplitude is involved in the function of the response characteristics of the system. The modalmetric sensor is frequently of lower sensitivity in comparison to the interferometer, but the output signal gives information directly about the existence of external effects of the mechanical fields. In the dual system, it is expected, therefore, that we can get the sensor response of the fact that interactions with higher sensitivity exist. So we are looking for features providing to improve the sensitivity of interferometric sensors for physical extortion. The study of systems, testing the configuration data and analysis results described later have to justify conclusions from the theoretical analysis. Because in the available literature, there is no analysis, therefore, presented studies were limited to basic research.

\section{Sensor systems examined}

Interferometer systems covered by the investigations were:

- Michelson interferometer,

- Mach-Zehnder interferometer,

- Sagnac interferometer.

Schematic diagrams of these systems are represented in Fig. 1, Fig. 2, Fig. 3.

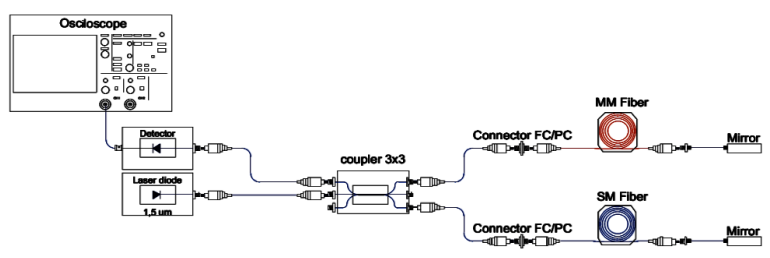

Fig. 1. Michelson interferometer in dual configuration.

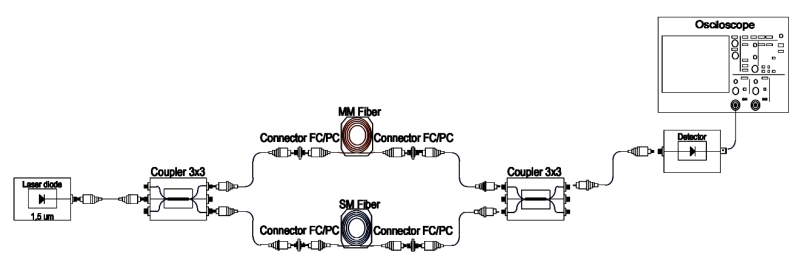

Fig. 2. Mach-Zehnder interferometer in dual configuration.

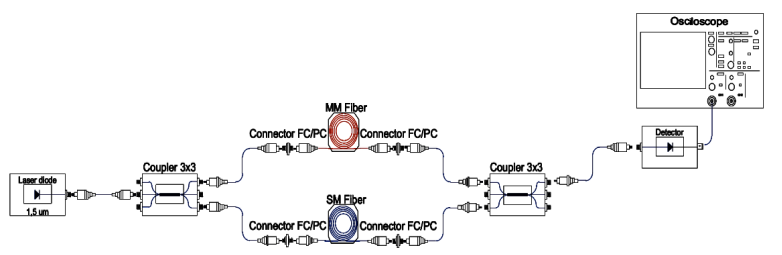

Fig. 3. Sagnac interferometer in dual configuration. 
As shown in the above diagrams in one of the branches of the track multimode fiber has been added. This is just the modalmetric sensor.

\section{Experimental details and results}

The tested systems were subjected to different types of disorders. First of all systems tested were classical and hybrid systems (with the modalmetric sensor). Disorders which were stimulated systems are:

- Harmonics mechanical forcing, the frequency of $1 \mathrm{~Hz}$,

- Harmonics mechanical forcing, the frequency of $50 \mathrm{~Hz}$,

- Accidental mechanical constraints, in the form of bending the fiber manually.

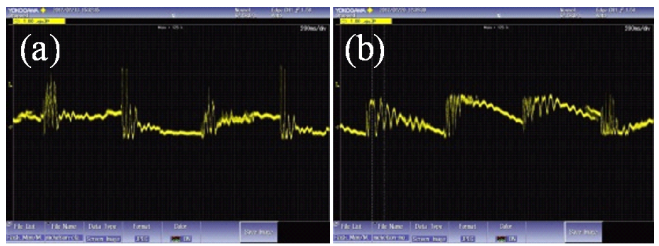

Fig. 4. Mechanical stab of the Michelson interferometer $1 \mathrm{~Hz}$ : (a) classic version, (b) dual version.

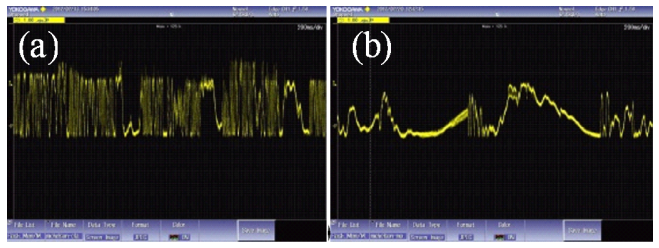

Fig. 5. Manual stab of the Michelson interferometer: (a) classic version, (b) dual version.

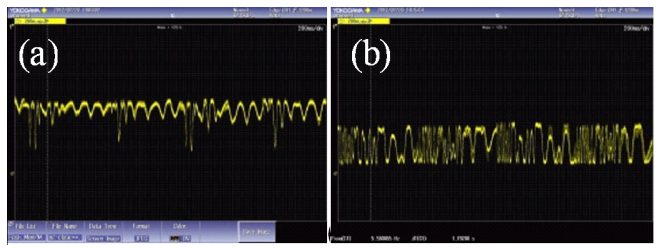

Fig. 6. Mechanical stab of the Mach-Zehnder interferometer $1 \mathrm{~Hz}$ : (a) classic version, (b) dual version.

Transmitter and receiver system consisted of a DFB laser-C5-1550 Series of multi quantum well (MQW) distributed feedback (DFB) and the receiving set built on a standard telecommunications diode, connected to an oscilloscope - DLM 2054, Yokogawa.

Reference system of mechanical disorders is controlled by the generator AFG3021 Tektronix.

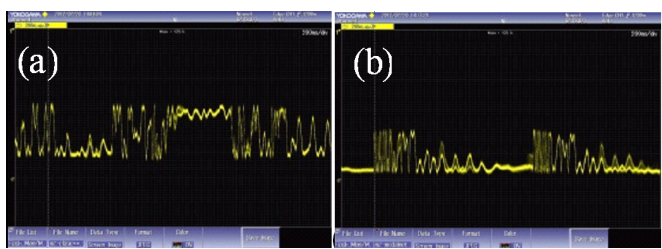

Fig. 7. Manual stab of the Mach-Zehnder interferometer: (a) classic version, (b) dual version.

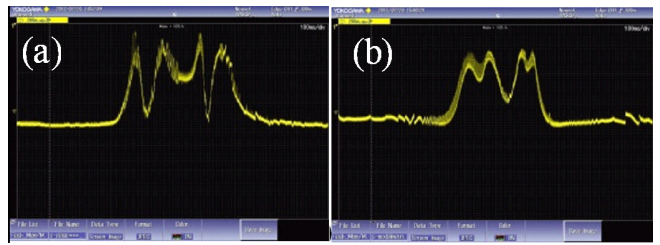

Fig. 8. Mechanical stab of a Sagnac interferometer: (a) classic version, (b) dual version.

First, the Michelson interferometer was tested. As the external field disorders there was used mechanical modulation of $1 \mathrm{~Hz}, 50 \mathrm{~Hz}$ and mechanical excitation in the form of manually bending the fiber section of the measuring arm. The most interesting results were obtained for the $1 \mathrm{~Hz}$ modulation and for manual disorder. The results obtained for $1 \mathrm{~Hz}$ modulation are shown in Fig. 4.

The results obtained for manual disorder are shown in Fig. 5.

Another tested interferometer was the Mach-Zehnder interferometer. As the external field disorders there was used mechanical modulation of $1 \mathrm{~Hz}, 50 \mathrm{~Hz}$ and mechanical excitation in the form of manually bending the fiber section of the measuring arm. The most interesting results were obtained for the $1 \mathrm{~Hz}$ modulation and for manual disorder. The results obtained for $1 \mathrm{~Hz}$ modulation are shown in Fig. 6.

The results obtained for manual disorder are shown in Fig. 7.

The last tested interferometer was the Sagnac interferometer. As the external field disorders there was used mechanical modulation of $1 \mathrm{~Hz}, 50 \mathrm{~Hz}$ and mechanical excitation by manually bending the fiber section of the measuring arm. The most interesting results were obtained for manual disorder and they are shown in Fig. 8.

\section{Conclusions}

For studies of hybrid dual configuration, modalmetric-interferometric, the results show improved response of the sensor signals directly affecting the fiber. The amplitude of the output signal is lost, because under the influence of pressure on multimode fiber, the product of interference disappears. One can say that the modalmetric system "modulates" the interferometric system, leading him to interference. The resulting signal is presented as a sudden descent of the signal amplitude to zero. This 
feature improves the known solutions of systems [4-9], allowing even easy location disorders of the sensors. The authors expect that as a result further research will reveal new features or improvement of the already known application solutions fiber optic interferometer sensors.

\section{References}

[1] M. Jedrzejewska-Szczerska, M. Gnyba, B.B. Kosmowski, Acta Phys. Pol. A 120, 621 (2011).

[2] T. Pustelny, J. Ignac-Nowicka, B. Jarzabek, A. Burian, Opt. Appl. 34, 551 (2004).

[3] M. Zyczkowski, B. Uziebło-Zyczkowska, L. Dziuda, K. Rózanowski, Proc. SPIE 7894, 413 (2011).
[4] M. Życzkowski, M. Kondrat, W. Ciurapiński, J. Phys. $I V$ : JP 129, 189 (2005).

[5] T. Pustelny, J. Ignac-Nowicka, Z. Opilski, Opt. Appl. 34, 249 (2004).

[6] M. Szustakowski, W.M. Ciurapinski, M. Życzkowski, Proc. SPIE 6736, 117 (2007).

[7] M. Szustakowski, M. Chojnacki, M. Życzkowski, N. Pałka, Opto-Electron. Rev. 9, 413 (2001).

[8] M. Życzkowski, W. Ciurapiński, M. Szustakowski, M. Kondrat, N. Pałka, Proc. SPIE 5566, 300 (2004).

[9] M. Kondrat, M. Szustakowski, A. Górka, N. Pałka, M. Życzkowski, S. Niżnik, Proc. SPIE 5611, 225 (2004). 